\title{
Leveraging the Twitch Platform and Gamification to Generate Home Audio Datasets
}

\author{
Nikolas Martelaro \\ HCI Institute \\ Carnegie Mellon University \\ Pittsburgh, PA, USA \\ Jingya Chen \\ Entertainment Technology Center \\ Carnegie Mellon University \\ Pittsburgh, PA, USA
}

\author{
Tarannum Lakdawala \\ HCI Institute \\ Carnegie Mellon University \\ Pittsburgh, PA, USA \\ Jessica Hammer \\ HCI Institute \\ Carnegie Mellon University \\ Pittsburgh, PA, USA
}

\begin{abstract}
Training AI systems requires large datasets. While there are a range of existing methods for collecting such data, such as paid work on crowdsourcing platforms, the strengths and weaknesses of each method leads us to believe that new, complementary methods are needed. The Polyphonic project contributes a novel method for collecting real-world data by piggybacking on game streaming communities such as Twitch, which capture over a trillion minutes of viewer attention a year. By embedding activities within the sociotechnical context of the stream, we can leverage some of this attention for data collection and processing. In this paper, we describe the design and implementation of a proof-of-concept system for collecting home audio data. We conducted a field study in four live streams and found that our proof-of-concept effectively supports data capture. We also contribute further design insights about stream-based data collection systems.
\end{abstract}

\section{CCS CONCEPTS}

- Human-centered computing $\rightarrow$ Interactive systems and tools; Collaborative and social computing.

\section{KEYWORDS}

live streaming, gamification, data collection, datasets, crowdwork

\section{ACM Reference Format:}

Nikolas Martelaro, Tarannum Lakdawala, Jingya Chen, and Jessica Hammer. 2021. Leveraging the Twitch Platform and Gamification to Generate Home Audio Datasets. In Designing Interactive Systems Conference 2021 (DIS '21), fune 28-fuly 2, 2021, Virtual Event, USA. ACM, New York, NY, USA, 18 pages. https://doi.org/10.1145/3461778.3462097

\section{INTRODUCTION}

Modern, data-driven science and AI systems are powered by access to large amounts of high-quality real-world data. Today, many of these projects utilize online crowdwork platforms to engage the

\section{(c) $(1)$}

This work is licensed under a Creative Commons Attribution-NoDerivs International 4.0 License.

DIS '21, fune 28-fuly 2, 2021, Virtual Event, USA

(c) 2021 Copyright held by the owner/author(s).

ACM ISBN 978-1-4503-8476-6/21/06.

https://doi.org/10.1145/3461778.3462097 public to collect and annotate data. For example, scores of paid crowdworkers label millions of images in the ImageNet database for computer vision applications [12], thousands of volunteer birdwatchers collect field observations of birds in their local environment for ornithology research [4], and hundreds of thousands of people play the computer game FoldIt to generate data on protein folding [8]. Each of these projects leverages a different strategy for engaging the public in data collection, namely, paid crowdwork [52], volunteer-based citizen science [36] and entertaining activities that yield useful data as a by-product of people's participation [57].

While all of these methods for collecting data have strengths, there are weaknesses that can limit the kind and amount of data that is collected. Paid crowdsourcing can incentivize people though financial compensation, but projects often alienate workers through underpayment and limited abilities to provide feedback on task design $[18,25]$. Volunteer-based experiences often leverage people's desire to contribute to worthy scientific causes, however, without recognition, people often drop out [38]. Entertaining activities such as games designed to generate data must first be entertaining enough to keep people engaged [57]. Without active engagement from participants, there is a dearth of data for researchers to use. Furthermore, all forms of crowdsourced data collection rely on managing positive relationships with data collectors [26, 48]. The challenges of various data collection strategies as well as the charge to develop positive relationships with data collectors prompt us to ask if there are other strategies for crowdsourced data collection?

To explore new methods for crowdsourced data collection and annotation, we look towards online live streaming communities as a way to promote engaging data collection activities that maintain positive relationships with data collectors. Online live streaming platforms, such as Twitch.tv, allow streamers to broadcast their game activities, while many viewers watch and engage with the streamer, typically through chat. Over time, these streams form informal communities [17] that already possess many of the factors for engaging groups of people online. Live streaming communities have strong long-term relationships built around shared activities $[17,62]$, promote donation to streamers [60] or charitable causes [45], leverage many forms of recognition to keep viewers engaged [60] and promote interaction among community peers [42]. Furthermore, live streams can attract a diverse range of people, leading to more diversity in the data we can collect. Given these properties, 
we believe that live stream communities can be used as a new arena for crowdsourced data collection.

To this end, we propose a novel method for combining the strengths of crowdwork with live streaming communities: creating a crowdsourced data collection and annotation service that runs in concert with existing activities on live streaming platforms. Our approach takes advantage of several key features of these live streaming platforms. First, streamers are trusted figures who have strong parasocial relationships with their communities [17]. Second, viewers seek attention from streamers [51]. Third, streamers desire new ways to engage their audiences in participatory, communitybuilding activities [62]. Taken together with the vast popularity of game streaming services [22] and long periods of viewer engagement [14], we see an opportunity to design data collection and annotation services that leverage the positive relationships and entertaining activities among live streaming communities.

In this paper, we introduce Polyphonic, a novel method for collecting real-world data by piggybacking on Twitch game streaming communities. We describe the design and implementation of our proof-of-concept system in the use case of collecting home audio data. Polyphonic is composed of 1) an interactive game that incorporates sounds collected from viewers in a streamer's community and provides recognition to those who collect sounds, 2) a mobile app where live stream viewers can collect and validate sounds from around their home, and 3) a server architecture to manage data collection, validation, and streamer events. We develop our prototype system around a set of design principles including respect for streamers, respect for viewers as data collectors, modularity, and sustainable interactions. We conducted a field study in four live streams and found that our system effectively supports data capture, allowing us to collect 212 sounds across 41 different categories around the home. These sessions revealed positive interactions, such as engaging more viewers in a stream than normal and promoting more community engagement, as well as challenges with our system design, such as the disconnect between collecting and validating sounds. Overall, our field studies suggest that our systems can engage live streaming communities in data collection activities while promoting positive relationship activities with data collectors. In summary, our contributions are:

- Design principles for volunteer-driven data collection systems within live streaming communities, which is a novel alternative to more traditional crowdwork data collection strategies.

- Polyphonic, a proof-of-concept system for engaging live streaming communities in home audio data collection that considers the social structure of live stream communities.

- Results of four field tests that suggest the potentials for data collection activities to integrate positively with live streams, as well as challenges and future research directions in this area.

\section{RELATED WORK}

Our research builds upon prior work on crowdsourced data collection and annotation strategies that leverage developing long-term positive relationships between data requesters and data collectors. We leverage best practices from and extend models from online crowdsourcing, citizen science initiatives, and relationship management among live streamers and their communities.

\subsection{Crowdsourced Data Collection and Annotation Strategies}

Crowdsourced data collection and annotation systems leverage different strategies for engaging people in data work including paid crowdwork, volunteer-based initiatives, and entertaining activities that yield useful data. Each of these strategies have different benefits and challenges related to engaging crowd participation and can support our work in developing new data collection strategies.

2.1.1 Paid Crowdwork. Paid crowdwork, where researchers pay people to complete short tasks on platforms such as Amazon Mechanical Turk, has become a standard method for data work due to its scalability and ease of deployment [52]. Often, crowdwork tasks focus on annotating data that is scraped from the web, such as the $14 \mathrm{M}$ images that comprise the ImageNet computer vision dataset $[39,53]$ and the $2 \mathrm{M}$ sounds derived from YouTube videos in the AudioSet dataset [16]. However, paid crowdwork can also be used for data collection tasks, such as in the Hollywood in Homes dataset where 267 crowdworkers created $10 \mathrm{~K}$ videos completing everyday activities in their homes [47]. With more people across the world having access to smartphones it is also becoming popular to conduct mobile crowdsourcing, where people use their mobile device with embedded sensors to capture data from the real world while also receiving an auxiliary form of income [13, 58, 65]. By recruiting crowdworkers for dataset collection, it is possible to capture more realistic and diverse data [47] than through scraping alone.

While paid crowdwork strategies for data collection and annotation can he highly scalable and cost effective, there are challenges that exist including underpayment of workers [18, 25], lack of worker input on tasks [26], and feelings of alienation due to poor communication between workers and requesters or with other workers [25, 31]. While these challenges can be overcome with good task design, good communication, and fair payment [26] the limitations of the method also suggest the need to explore methods for crowdsourced data work that provide different benefits, attract different communities, and afford different opportunities than paid crowdwork.

2.1.2 Volunteer-based Crowdwork. Volunteer-based crowdsourcing methods rely on non-financial incentives and benefits to recruit volunteers to conduct data work [26]. Volunteer-based activities have been adopted across the sciences in the form of citizen science projects ranging from contributing common sense assertions to an AI database [50] to classifying images of galaxies [28] to bird observation [54]. The popularity and effectiveness of data collection and annotation through citizen science has led to the growth of online citizen science platforms similar to paid crowdwork platforms, such as Zooniverse [49] and CitSci.org [59]. Volunteer work can produce similar quality work [30] and can provide benefits to crowdworkers who are not fully motivated by financial incentives [26], such as addressing people's desires to contribute to science, make a positive impact, and connect with others in the community [2, 20,37]. 
Studies looking at understanding how to engage the public in participating in science [24] can be useful for designing new data collection and engagement strategies. Research on people's motivations for participating in citizen science data collection has shown that volunteers often participate because they want to support scientific endeavours. Shirk et al. [46] define five levels of citizen science projects that define the different models by which scientists and the public engage with each other. Among these classifications, the majority of projects are contributory (volunteers contribute data), however, some projects are collaborative (volunteers contribute data and influence aspects of research design) or even co-created (volunteers are involved in all aspects of research design). Further work by Phillips et al. [36] proposes a 'Dimensions of Engagement' framework for citizen science that incorporates cognitive, affective, social, and behavioral motivators for participation. Specifically, it is recommended to design features that support social interaction among citizen science volunteers as it is a key motivator for sustained engagement, as found by Curtis [9]. In our work, we leverage the inherent social interactions that occur in live streaming communities to motivate volunteering.

2.1.3 Entertaining Activities that yield useful data. Entertaining activities that yield useful data consist of activities that are not explicitly about data collection or annotation, but that yield new data or labels for use by a research team. Games are a primary activity that fit this model of engaging data workers, for example the ESP Game by Von Ahn \& Dabbish [56] where people labeled images by trying to predict what other online players are thinking as labels. These kinds of games, known as Games with a Purpose (GWAP), are specifically designed to be enjoyable for the players and to yield useful data or analyses that are easy for people but challenging for computers to determine [57]. Within the sciences, a popular GWAP is FoldIt, where players solve puzzles that ultimately simulate how proteins fold [8]. Research on FoldIt players by Curtis [9] found that regular contributors to the game were motivated by the intellectual challenge of the puzzles. Similar to other findings on motivation in citizen science, players were also motivated by contributing to scientific research.

Beyond explicitly designed GWAP activities, other activities can serve the role of providing enjoyment to people and useful scientific data. Members of the FoldIt community reported that social interaction with others outside of the game was a significant motivator in their continued engagement [9]. As another example, many citizen scientists who participate in environmental and ecological studies consider getting outside and enjoying nature as a key motivator to volunteer $[2,20]$. These alternative motivators suggest that there are other kinds of entertaining activities that can be designed to also yield useful data. Our work looks to leverage various kinds of entertaining activities that occur in Twitch live streams and outside of the live stream to motivate and engage volunteers in data collection and annotation.

\subsection{Participatory communities on live streaming platforms}

Game streaming platforms, such as Twitch.tv and Youtube.live, allow streamers to share a live audio-visual stream, which typically includes gameplay and a video of themselves. Viewers watch, and can participate in synchronous text chat. Finally, moderators help manage the chat and mediate between the streamer and their community. While there are a number of game streaming platforms in existence, Twitch.tv is the largest, with over 3.8 million unique streamers and 1.4 million concurrent viewers [22] and a strong research base [19]. Prior work has also shown Twitch to be a digital third place where communities are formed and participate in shared social interactions [17].

Live streams occupy a complicated space that integrates paid work, volunteering, and entertainment, qualities that we can leverage for our work developing data new kinds of collection systems. Streamers monetize their channels using a variety of strategies [23]. For example, some streamers ask viewers for one-time donations, then reward them with verbal thanks and automatic screen graphics on the stream. Streamers may also receive sponsorship from advertisers or monthly subscription payment from viewers. At the same time, many Twitch communities pride themselves on their civic-mindedness. On "charity streams," streamers play to raise money for a cause [32]. Streaming communities can also be leveraged for microvolunteering [40], where short breaks from the stream are used for socially conscious microtasks. Finally, entertainment is a major motivation for stream viewing [51]. Viewers enjoy watching the game being played and fulfill social needs through the relationships they develop with the streamer and with the viewing community [21].

As the above suggests, relationship management is key to successful streaming. The streamer plays a key role in fostering community by developing parasocial relationships with viewers [3]. These relationships are a strong predictor of financial support for live streamers [62] and of repeated viewing of streams [27]. A sense of community among viewers is also important and supports long-term engagement in stream channels $[21,51]$ as evidenced by viewers wanting to be a part of the live engagement [60] and where communities even form their own language and subcultures [33].

One common strategy for relationship management in game streams is fostering engagement between the streamer and viewers, as well as within the viewing community, through shared activities $[17,21,60,62]$. For example, live streamed audience participation games allow viewers to take part in the streamer's gameplay [43]. Some viewers value the resulting attention from the streamer, while others enjoy the influence they have within the viewer community [43]. Another common strategy is streamer recognition, where streamers elevate viewer activity. Recognizing donors with an onstream shout-out is one type of streamer recognition [62], but streamers can also discuss activity they see in the chat, talk back to comments made by specific viewers, or invite valued community members to play with them live [60].

Finally, live streaming platforms incorporate a surprising range of streamed activities. Within the game context, streamers might showcase a variety of games [35], teach techniques in a game where they are an expert [34], participate in esports [61], or play a game to raise money for charity [45]. However, many streams focus on activities other than gameplay. For example, "creative" streams include everything from live game development feeds to craft-alongs, while talk shows and streams about daily life are also popular [14].

Taken together, these features of live streaming platforms make them an excellent target for designing new types of crowdsourced 
data collection relationships. Stream communities already have strong long-term relationships built around shared activities; activities beyond strictly gameplay are welcome and appreciated; recognition is common and supported through social and technological systems; and we can draw on a range of motivations for crowdsourced data collection, including paid, volunteer, and entertainment approaches. To develop well functioning and valuable data collection systems, we have to meet the needs of streamers, viewers, and other stakeholders.

\subsection{Audio Data Collection}

To develop our stream-based data collection systems, we have chosen to build a proof-of-concept system for collecting audio data from around the home. Home audio data presents an interesting challenge for data collection for a number of reasons. First, clear audio data of different sounds around the home can be hard to scrape from the open web. Unlike image-based datasets such as ImageNet [12] where millions of images can be downloaded from the web, audio data often exists within other media such as videos. The AudioSet project [16] compiles a dataset of 2.1 million labeled sounds by pulling audio data from short clips in YouTube videos. These sound clips were then labeled with categories such as electric toothbrush, music, speech, or lawn mower by crowdsourced human annotators. Similarly, VGGSound [7] also scrapes YouTube videos for audio, but labels images based on computer vision of what is in the video. While each of these datasets contain sounds related to the home, many clips contain background noise such as music or people speaking, reducing the quality of the specific audio data. To better enable machine learning applications, it may be better to collect clear audio data without extensive background noise of different objects in the home.

Second, home audio data in particular is collected in people's private spaces and thus requires more interaction and consent from people to record the sounds. Prior work has developed intelligent microphone systems that constantly listen to sounds in the home and then ask users what a unique sound is [64]. This methods has the benefit of learning sounds from fewer examples and leveraging users to label data rather than requiring groups of crowdworkers to do so. However, this strategy for data collection and annotation requires always-on audio capture, something that some people may be uncomfortable with as there may be times when they prefer audio not be recorded. Additionally, such devices are typically fixed in specific areas of the home, limiting people's ability to capture sounds around the home such as the different windows on upper and lower floors, or doors in the garage, living room, and bedroom.

To address these challenges, we take inspiration from citizen science and dataset creation projects that engage people in actively collecting data [36, 46], such as the Hollywood in Homes project [16] where crowdworkers film themselves doing specific activities around their home to build a dataset for visual activity recognition. By recruiting people to engage directly in the data collection, annotation, and validation process, we can collect a more focused dataset of sounds specific to the home. We can also better control the quality of data by having people record audio without background noise and by capturing data from the different locations around their home. Finally, by engaging volunteers directly in data collection, we can minimize privacy concerns as the data collectors explicitly record audio that they intend to annotate and share. We attend to these issues in the design of our proof-of-concept to collect home audio data through live stream activities.

\section{DESIGN}

In this section, we describe the design and development of our proof-of-concept system. First, we identify the design principles we used as constraints, and explain why they were chosen. Next, we describe our design process. Finally, we explain the system itself, both at a technical level and how it operates in the social context of game streaming platforms.

\subsection{Design Principles}

The principles that guide our design were developed based on the literature on volunteer-based data collection activities and participatory communities on live streaming platforms. We used Joseph Seering's Twitch Research Bibliography [41] to review all the literature on Twitch published at the time of our design work. We did an affinity diagramming process with the papers to develop themes. These themes were then better formed and validated during our Research-through-Design process and in conversations with streamers. We note that our design principles were developed based on a very specific context-collecting audio data collection in the home. Rather than attempt to generalize backwards, we are exposing these principles. We believe that our proof-or-concept can generalize to other types of active data collection tasks, but discuss within our specific context.

3.1.1 Respect streamers' needs. Streamers are the ones who decide what activities take place on-stream. If we are asking streamers to share their platforms with us for data collection efforts, the design must serve them. We do not want our work to be limited to streamers who are already interested in AI, sound data, or crowdsourced data collection. Instead, we design for their stream-based needs.

As the literature shows, streamers value viewer engagement $[21,51]$. Engaged viewers are more likely to return to the stream [21] and to support the streamer financially [23, 62]. Streamers already deploy a range of techniques to manage their audiences [60]. If our system provides audience engagement opportunities, then we are helping streamers accomplish something they already value.

Additionally, streaming is taxing work that incorporates both gameplay and improvisational performance $[55,63]$. Streamers are often reluctant to take breaks because they believe that audience engagement is reduced when they step away from the screen, for example, to get food or use the restroom. We therefore added the design goal of providing activities for viewers that would allow the streamer to take a quick break, or that could even be done away from the stream.

3.1.2 Respect viewers' contributions. Stream viewers could be construed as passive consumers of content. However, the literature shows that stream viewers can be active partners in constructing the culture and community of a stream [17]. Viewers participate in chat, interact with bots, and can even participate in streamers' gameplay $[43,61]$. Our design therefore does not need to move 
viewers from passive to active, but rather to respect and leverage the ways they already contribute to the community.

Our design also needs to respect viewers' motivations in the Twitch environment. In the same way that you wouldn't pay your mother to wash your share of the dishes, viewers' motivations cannot simply be replaced with financial incentives. Viewers come to Twitch to be entertained cognitively and emotionally, to interact with a community, and to engage with a streamer [51]. For example, many viewers are highly motivated by being recognized by the streamer, e.g. by having their names read on screen. Our design should give them opportunities to better realize a range of motivations through participating in the data collection process.

Our design also needs to respect user's privacy and willingness to volunteer. Audio data collection in the home makes these aspects more important. For example, viewers may accidentally capture private sounds, such as people talking, while collecting requested sounds like a dishwasher running. Viewers may also 'misfire' and fail to capture the sounds they intend. The environment might be too noisy, or the sound could be fleeting and they simply missed their window. It is important to have strong privacy safeguards regardless of the type of data being collected; for audio data, we want to avoid capturing sounds with people speaking or revealing personally identifiable information and we want data collectors to know exactly what they are sharing with the larger data collection system.

3.1.3 Build a modular system. In our initial work on audio data collection, we identified four possible crowdsourced tasks: capture, segmentation, labeling, and validation. We also identified different stakeholders (viewers, streamers, moderators, researchers) and different phases of streaming (before live streaming, during the live stream, and after the live stream). We needed to decide how we conceptualize the relationship between tasks, stakeholders, and stream phases.

We decided that focusing on the social structure of the system was the best strategy. Rather than trying to redirect the social practices of this large and vibrant community, we could leverage the social practices to accomplish specific parts of our goal. For example, during the stream, streamers already reward viewers with social attention [60-62]. We could therefore repurpose that existing behavior to support a crowdsourced data collection task. Additionally, considering different aspects of a stream would allow us to better manage the audio data collection process; interactions for data collection, annotation, and validation could be designed for different phases of a stream such as pre-stream activity, in-game interactions, and in-chat interactions. By designing new interactions to slot into our proof-of-concept as modular components each with a clear purpose and clear value to the stakeholders, we could emphasize different parts of the audio data collection process and the social interactions that occur within streaming communities. We described this internally as a "suite of games" approach, although we quickly discovered that other interactions (e.g. social recognition, validation) could work as well.

For our initial designs, we focused on two stakeholder groups: streamers and viewers. Not all streams have moderators, and researchers do not need to be present during data collection, thus we did not focus on moderation in this work. We also focused on two phases: before-stream and during-stream. Before a stream, streamers publicize the upcoming stream, plan their activities, and connect with viewers on external services such as Discord. During the stream, they play games and interact with their audience. Meanwhile, viewers engage in chat and discuss their viewing experiences. Because most streamers build an ongoing audience, we construed "after-stream" activities as simply being "before" the next stream.

3.1.4 Build a sustainable system. One advantage of working with game streaming communities is that the viewers spend on average 100 minutes per day streaming [21]. Streamers typically stream on a regular basis, and work to cultivate long-term, engaged audiences. These features of the streaming ecosystem allow the design of systems for long-term engagement with data collection. Rather than making data collection one-off and/or transactional, as might be the case on crowdwork platforms like Mechanical Turk, we seek to design a data collection system that repeatedly and relationally engages participants.

To sustain long-term engagement, the game elements of the system must focus on replayability. Producing enough hand-crafted game content for long-term play is, at this point, out of scope for a research team. That requirement in turn eliminates entire game genres (e.g. single-player RPGs, many types of puzzle games). It instead places the game in one of two design spaces: games with procedurally generated content, or multiplayer games where the other players' choices provide depth and richness.

The other component of long-term engagement is providing meaningful recognition. Prior work shows that recognition-being appreciated and valued for their contributions-is critical to making the social engagement with a data collection system sustainable. Recognition is not a one-size-fits-all experience, both in terms of what people are looking for and in terms of what different components of a modular system can provide. We therefore aim to design recognition opportunities in each component of our proofof-concept. This design choice allows us to recognize people who may only participate in some modules of the experience. It also allows us to draw recognition opportunities out of the material at hand, such as by leveraging opportunities for the streamer to connect with their audience as described above.

\subsection{Design Methods}

We began our design process by creating games that could work for audio data collection using GWAP principles. Early methods involved a structured ideation process. A team of eight student designers generated over 250 early game concepts. The team then used an affinity diagramming process to extract themes from the game concepts. These themes were then used to create a set of fifteen fleshed-out design sketches, which were robust enough that they could be considered for prototyping. Each sketch focused on one audio data collection activity (e.g. data validation), but was allowed to include other data collection activities if the game design required it. This type of sketching and iteration process is typical for exploratory game design processes, for example as described in [29].

To evaluate our sketches, we collaborated with audio data experts to create a set of metrics for the sound collection and labeling process. The core metrics were quality, accuracy, productivity, 
efficiency, and playability. Metrics were prioritized differently for different data collection tasks. Quality was a high priority for sound capture, while accuracy was most important for labeling. Productivity (measuring tasks completed per game loop) and efficiency (measuring tasks per time unit) were used to filter out ideas where the game development effort would outweigh the data collection value. Finally, each game concept was rated for playability- to what extent did we think the game would be fun to play (e.g. for task completion), and to what extent did we think it would be fun to watch (e.g. to encourage viewer engagement). If games were not fun on both axes, they were removed from consideration. Each designer independently rated each game sketch on a scale of 1-5 in each area. The team then discussed the ratings until all designers were in agreement. As described above, games that did not meet minimum productivity, efficiency, and playability standards were removed from the list. This strategy ensured that the prototype pool contained only games that were a) feasible to make and b) had lots of fun potential. From within this group, ratings were then used to select games to move forward, with the highest emphasis being placed on quality and accuracy.

To understand how we might integrate our games with the Twitch platform, we took a service design approach [66]. We created journey maps for both Twitch viewers and streamers, including points of contact with our system (game and app), and identified their frontstage and backstage activities. Unlike a traditional service design approach, we considered Twitch streamers and viewers as co-creators of the experience; each party had their own frontstage and backstage experiences, as well as distinct things to offer and potentially distinct goals.

We recruited a student game development team to create a working game prototype. The students were given our top three game sketches, and we worked with them to incorporate our insights into the Twitch ecosystem into their final design idea. The team then created a mobile data collection app, a Unity game called Rolling Rhapsody, a streamer dashboard, and a database that supported communication between the other components. Our playcentric iterative development process [15] focused on finding the fun in the gameplay, making the mobile app easy to use, and validating the functioning of the underlying platform. Bootstrapping playtests that required a) viewer-submitted audio data and b) engagement from streamers was challenging. However, we were able to playtest effectively using a combination of techniques, including using a database of sample sounds gathered by the team, using WoZ techniques [10] to let early testers collect data by emailing team members audio recordings, and having a confederate run sample streams. While we learned a great deal from this iterative design process, we do not consider these to be as useful as field tests for our proof-of-concept, as the researchers and the design team were heavily involved in the process. For descriptions of our field tests, see Section 4 below.

\subsection{Polyphonic Proof-of-Concept Description}

We now describe the components of the Polyphonic proof-of-concept system. Polyphonic incorporates a mobile data collection app where viewers can collect audio from around their home and then donate these audio clips to streams, a process for validating the sound labels, a game that incorporates the collected sounds into the gameplay and recognizes viewers for their contributions, a dashboard for streamers to manage the live stream events and data requests, and database systems that coordinates sound collection and viewer data collection.

The general flow of interaction (Figure 1) in the proof-of-concept is:

(1) A streamer creates an event using the streamer dashboard and requests sounds to be collected for the event.

(2) The streamer then advertises their event to their viewer community and asks viewers to download the data collection app.

(3) Viewers log into the app using their Twitch ID, pick a character, select the streamer's event, and collect sounds from around their home to donate to the event.

(4) Viewers then use the validation interface to confirm that sounds are labeled correctly.

(5) During the live stream event, validated sounds are used in a game where the audio samples are collected by the streamer. Sounds are played in the game and the viewer who donated the sound is recognized on screen.

(6) After the game, a leaderboard shows the viewers who donated the most sounds.

Each piece of the system is modular and can be redesigned on its own. As described in the Design section, this modularity allows for our system to be designed for specific aspects of the data collection and annotation process, and to fit with the varied social aspects of live streams such as participating in games, donating to streamers and receiving recognition.

3.3.1 Mobile app for data capture and validation. In our proof-ofconcept, viewers are responsible for data capture and validation using a mobile app that is downloaded to their personal device, shown in Figure 2 (Left). Viewers log into the app using their Twitch ID so that they can later receive proper acknowledgement for their contributions during the live game stream.

Viewers record sounds onto their mobile device and tag the sound with a descriptive label (e.g. "electric toothbrush") (Figure 2 Left - Screen 1). Because recording takes place on a mobile device, viewers can carry their devices around the house to record sounds in different rooms. Viewers might be asked to collect specific sounds, but they can also notice a sound in their environment and decide to capture it, as they are likely to have their phone with them. All sounds are collected into a personal sound library, which allows the viewer to delete sounds that they do not wish to share. Before donating a sound to the database, viewers must consent to sharing the audio by checking 'Agree to share.' This ensures that viewers control every sound they share with the larger dataset that can be used on the live stream.

When a piece of audio data is captured, it is associated with a streamer event (see below). The sound will count toward that streamer's channel statistics, and has an increased chance of being used on-stream by that streamer. The streamer can also issue challenges to viewers to capture particular sounds for their event that viewers can focus on collecting. 


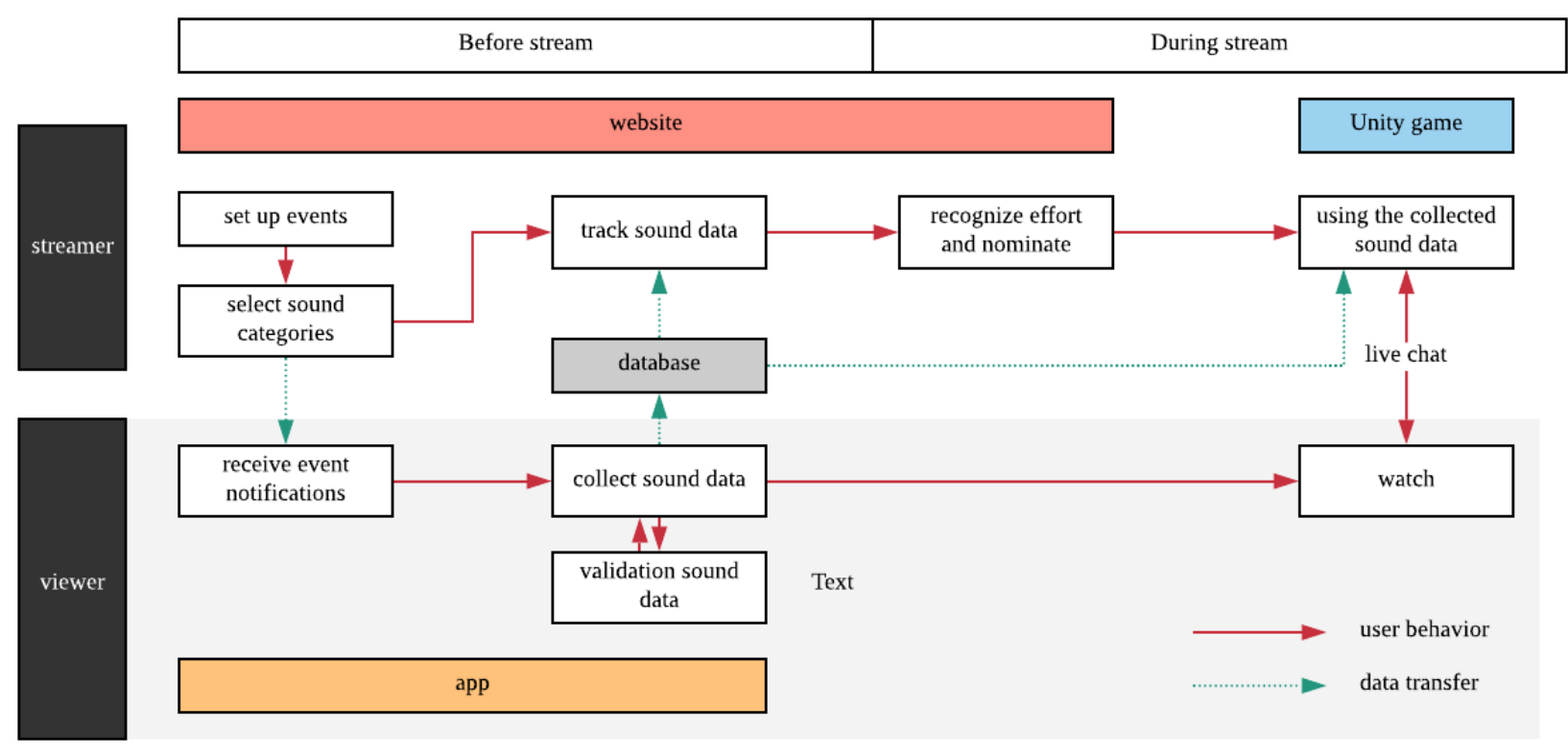

Figure 1: Interactions flows for events using Polyphonic to collect data. Streamers set up events, request audio from viewers, hear collected audio in the Rolling Rhapsody game, and recognize viewers for their contributions. Viewers receive event notifications, collect and validate sounds data from the home, and watch the stream and engage with the streamer.

When capture is complete, sounds are uploaded to the system database, where they can be downloaded by other viewers for validation and quality control. During the validation process, viewers are asked whether the sound they are hearing matches the label it was assigned (Figure 2 Left - Screen 2) by answering "Is this the sound of a(n) X?" with either 'yes' or 'no.' Viewers could also select 'unsure' if the sound was not clear enough, for example due to too much background noise or poor volume level. Once sounds were confirmed by at least three viewers, the sound is marked as usable in the game. If the sound is mislabeled, or if viewers report it as inaccurate, the sound cannot be used on-stream. Furthermore, users can report inappropriate sounds such as those with speech or those with malicious content using the 'Report' button, removing them from the pool of sounds used during the live stream and helping us to avoid violating the Twitch terms of service.

3.3.2 Game with audio and on-stream rewards. To engage both streamers and viewers with sound capture, we created a game to be played on-stream, where the streamer's gameplay integrates the viewer-collected sounds. Our game is called Rolling Rhapsody. In Rolling Rhapsody, the streamer controls a ball-shaped avatar who rolls around the game space collecting objects. Before the game, each viewer chooses an object (a tiny cactus, a purple mushroom) to represent their sounds. Then, at the start of the game, objects are randomly generated based on the validated sound data. Viewers with more validated sounds in the database have a higher chance of having their objects appear in the game. When the streamer collects a sound by rolling over an object, the sound associated with that object plays for everyone to hear. A written message thanking the viewer who collected that sound appears on-stream, visible to the entire community. The streamer has the opportunity to react to the sound, for example by laughing, and can verbally amplify the written thank-you statement. During each round of the game, the streamer receives a series of missions, such as "Collect three of the same type of object." The streamer must complete as many missions as they can in three minutes.

Objects in each level are randomized, pulling from all validated sounds that the viewers have collectively donated to the stream. If the viewers have not collected enough sounds to populate the level, then any validated sound in the dataset can be used. Object placement within the level and mission presentation are randomized as well, to allow replayability.

At the end of each play session, the game displays data about whose sounds were collected by the streamer. This encourages viewers to try to get the streamer to pick up the objects that are most likely to represent sounds that they contributed. Unlike statistical data about viewer contributions (see below), this data is refreshed each time the streamer plays the game. If a viewer's sounds are not collected in one three-minute playthrough, they may be collected in the next one. This dynamic encourages players to ask the streamer to play the game just one more time, so that they can share in the social recognition.

3.3.3 Streamer dashboard. Just as viewers manage their participation in a mobile app, streamers manage theirs in a web interface and $\log$ in with their Twitch ID. We chose a web interface because 


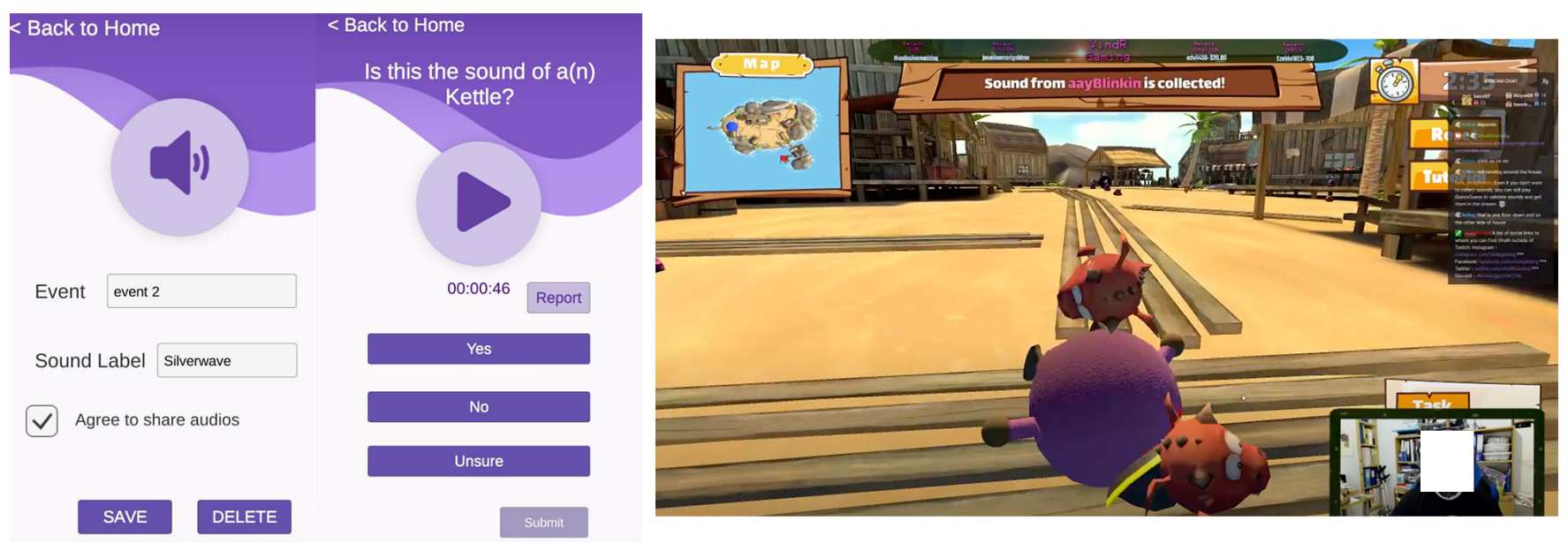

Figure 2: (Left) Mobile data collection app screens. Screen one shows the sound collection, label, and consent checkbox interface. Screen two shows the label validation interface where viewers confirms other's sound labels and quality before it is used in the game. (Right) Rolling Rhapsody game during a live stream. As a streamer rolls over an avatar the donated sound from a viewer is played. The contributing viewer's TwitchID is displayed at the top of the screen to give recognition.

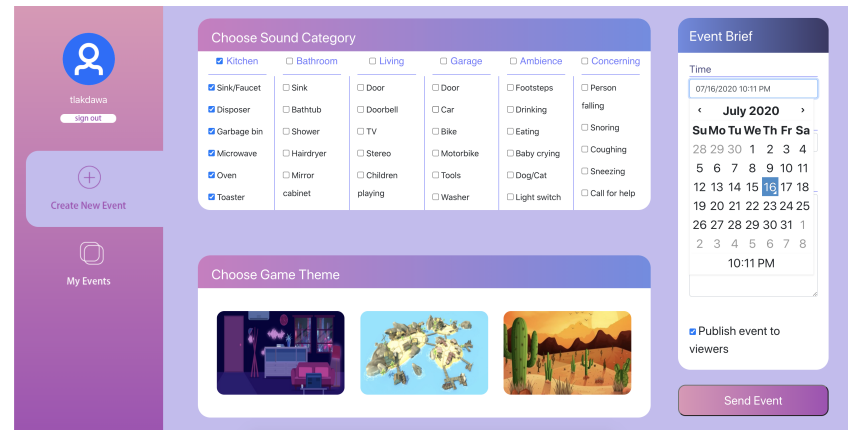

Figure 3: Streamer dashboard where the streamer can create a new event, select sound categories for their community to collect, and choose a game world to play during the stream.

streamers are typically sitting at a computer when sharing their video, and can therefore easily share it to their stream if desired.

One key role for streamers is to create game events, where viewers can donate sounds. Streamers are most effective at collecting an audience when they plan events ahead of time and publicize when they will be online [60]. Our event creator therefore includes the date and time at which the streamer will be using Polyphonic on-stream. The streamer can also select which sounds they would like their viewers to collect for them. The selection form is prepopulated with home audio data collection sounds. Streamers can create funny themed events using the event creator (e.g. all bathroom sounds), or they can ask their viewers to collect sounds that are particularly scarce in the system as a whole.

The streamer interface also allows the streamer to track and review sound collection statistics. These statistics are compiled on a per-event basis, and are nicely formatted for sharing with their viewers. The streamer can share key statistics about both individual and community participation on-stream, to reward individual contributors and the community as a whole. For example, the streamer can share a screen that ranks participants by the total number of sounds they uploaded, and gives special recognition to the top three sound collectors. A second screen shows the community's favorite sound (e.g. what was most often collected) and a word-cloud of the community's overall participation in the event. Finally, the streamer can see and share summative statistics about their channel's overall contribution to the larger data collection enterprise, independent of any given event.

3.3.4 Database for centralizing sounds. Our back-end database stores all sounds and game information. Sounds are stored with information about the sound contributor and the state of the sound's validation in the system. A future game could pull all non-validated sounds to share with the viewers to have them validated. Like our viewer and streamer interfaces, the server integrates with the Twitch platform to enable user authentication and (with permission) profile information.

We implemented our proof-of-concept using Unity, node.js, MongoDB, and the Twitch API. Data was passed between system components using web APIs, with the exception of live synchronous gameplay which used WebSocket communication.

\section{FIELD TESTS}

To test if the Polyphonic proof-of-concept could engage streamers and viewers in collecting audio data, we conducted a series of field tests with four streamers and their associated viewer communities. During these tests, streamers would use Polyphonic to set up a data collection and audio gameplay event. Streamers would then recruit their viewers to download the data collection app and collect audio samples from around their homes and validate samples from other members of the community. Then, during the stream, the streamer would play Rolling Rhapsody. Sounds from their viewers would be heard in the game and viewers would be recognized on-screen 
and through streamer call outs. We observed these sessions and collected data on viewer engagement and streamer interactions to understand how well the Polyphonic proof-of-concept supported collecting audio data and met the needs of streamers and viewers.

\subsection{Streamer Recruitment}

Suitable streamers were seen as those with smaller communities between 6 and 1000 live viewers and who often focus on building relationships with their viewers [14], are variety streamers [55] who frequently test new games with some degree of audience participation in the form of them asking game-related questions to their viewers, and stream in English.

In order to recruit streamers willing to test the proof-of-concept with their audiences, we reached out to potential candidates through Twitch by using the contact methods listed on their Twitch pages and by contacting their moderators through chat in a live stream. Otherwise, Twitch streamers who have worked with the lab previously were contacted and asked if they are interested in streaming again. We had a total of three streamers participate over four sessions. Streamers were encouraged to stream for as long as they wanted with the longest session lasting five hours and twenty five minutes.

\subsection{Streamers \& viewers}

We recruited three streamers to test our proof-of-concept across four live stream sessions (summarized in Table 1).

- Streamer 1 is a gaming streamer with 600 followers and 30 average viewers. Their stream lasted 1 hours and 27 minutes.

- Streamer 2 is a gaming streamer with 71 followers and an average viewership of 3 people on live streams. Their stream lasted 1 hour. Unfortunately, there were numerous technical issues during this stream and data collection was limited, though their feedback was useful for improving the technical aspects of the game and app.

- Streamer 3 is a makers \& crafts streamer with 2,900 followers and an average viewership of 50 people on their live streams. Their stream lasted 5 hours 25 minutes. This streamer typically streamed themselves doing crochet or other crafts. In our test, the streamer used Polyphonic and played Rolling Rhapsody during breaks between crocheting.

- Streamer 4 was the same streamer as streamer 1. During their second streaming session they had 771 followers and an average viewership of 12 people on their live streams. This second stream test lasted 1 hour 53 minutes and was dedicated to playing the game.

While Streamer 2's average viewership was lower than our intended target, we opted to include them in our field tests to test our technical systems and to see if such an activity could engage more viewers. Unfortunately, due to technical issues, we ultimately did not collect any data from this session and so only report on the results for Streams 1, 3 and 4.

To test replayability, we engaged out first streamer again as Streamer 4. Through testing with the same streamer, we aimed to observe how streamers and viewers would respond to and adapt the system during a second session.

\subsection{Procedures}

Multiple days before the stream, streamers signed consent forms agreeing to participate in the playtest and have their steam recorded. Streamers were compensated at a rate of \$10/hour via an Amazon gift card. Streamers were given a standard set of instructions for how to use the system and suggestions for how to conduct the stream such as requesting different sound categories and recognizing audience members for their contributions. A research team member explained the Polyphonic system including the data collection app and the Rolling Rhapsody game. All streamers were encouraged to set up the stream event in advance although some streamers chose to set up their events during the live stream and in front of their audience. Streamers were also encouraged to send out messages to their community with download links to the data collection mobile app. While setting up the sound collection event, streamers were able to choose what sound categories they wanted their viewers to collect sounds for.

At the start of the live stream test session, streamers encouraged their viewers to download the app, join their event, and contribute sounds to the game. Viewers downloaded either the Android or iOS application on their device. Viewers electronically consented to participating in our research before signing in with their Twitch ID so that we could track viewer's sound donation and so they could be recognized in the game. Viewers selected their streamer's event and then collected sounds around their home. Before uploading each sound to our server, the viewer provided consent to have the sound clip uploaded and entered into the database.

Once the streamer deemed that enough time had passed for their audience to submit sounds, they played Rolling Rhapsody on the stream. As they played, viewer's Twitch ID's were shown on screen when their sounds were collected. Viewers engaged with the streamer and other viewers through the Twitch chat feed. Our team was also available in the chat feed to provide technical support to the streamers and viewers. Streamers were allowed to play the game as many times as they wished. At the end of the stream event, streamers filled out an online questionnaire to provide feedback on the Polyphonic system, the game Rolling Rhapsody, and their interactions with their viewers during the stream.

\subsection{Field Test Data Collection}

From each stream we collected data as follows: the duration of stream, the number of sounds collected by the audience, the number of sounds validated by the audience, the maximum number of live viewers during the stream, the number of active viewers who interacted with the streamer in the chat, the number of categories sounds were collected for by the viewers who participated, and various categories of the collected sounds. All data about the stream such as peak number of live viewers and active viewers in chat were recorded by one of the research team members observing the stream live. Data on sounds collected and validated by viewers was collected on our database server. We also recorded the streams to log what actions the streamers took while interacting with their audience and to capture what occurred as they played Rolling Rhapsody. 
Table 1: Streamer characteristics and data collected from each stream.

\begin{tabular}{l|llll}
\hline Streamer & $\mathbf{1}^{*}$ & $\mathbf{2}$ & $\mathbf{3}$ & $\mathbf{4}^{*}$ \\
\hline Community size & 600 & 71 & 2900 & 771 \\
Time zone & CET (GMT +1:00) & EST (UTC -5:00) & EET (UTC +2:00) & CET (GMT +1:00) \\
Stream topic & Gaming & Gaming & Makers \& Crafters & Gaming \\
Average viewers & 30 & 3 & 50 & 12 \\
Length of stream (hrs) & $1: 27$ & $1: 00$ & $5: 25$ & $1: 53$ \\
Active viewers during stream & 12 & 2 & 9 & 3 \\
Gameplay sessions & 3 & - & 5 & 3 \\
Sounds collected & 110 & - & 82 & 20 \\
Sounds validated & $-* *$ & - & 82 & 3 \\
\hline
\end{tabular}

* Streamer 1 conducted the field test twice, again as Streamer 4, to test replayability. ${ }^{* *}$ No validation data was collected during this field test

We collected qualitative feedback from the streamer in a postsession questionnaire. The questionnaire specifically asked streamers to rate the experience of setting up a stream event, the appeal of the Rolling Rhapsody game concept, the data submission/validation process, the experience for their viewers, and how likely they would be to stream the game again. All questions were rated on a scale of 1 (low) to 5 (high). In addition, streamers were asked to provide subjective feedback on the game concept, how engaging the game was in practice, and any additional comments or questions they wanted to share with the research team.

\section{RESULTS}

\subsection{System Functionality}

Our field tests demonstrate that the Polyphonic proof-of-concept was able to support data collection by engaging viewers of live streams. We successfully captured and validated data during streams 1, 3 and 4. Streamer 2's test was unfortunately plagued by technical issues arising from a Unity software update. This issue was addressed live during the stream and some viewers were able to capture some sounds, but no sounds were validated and used in the game. We thus include only data from streams 1, 3, and 4 in our numerical results. Across the three functional field tests, we collected 212 sounds in 41 categories from 26 viewers. Figure 4 shows the number of sounds collected across each category.

Although we initially assumed that streamers would encourage their viewers to collect sounds before the event, streamers preferred introducing their audience to the app during the stream and then having viewers collect sounds as part of the stream activities. The majority of viewers were able to download the mobile app during the stream, set up their account, join the streamer's specific event, collect sounds, and upload them, all during the stream. During the steam we asked viewers to report any issue they had. Our observing research team members only had one viewer report a technical issue with the app. This being said, although many viewers were willing to use the app, some viewers opted out of participating, citing trust issues because the app was not available through the Apple App Store or Google Play Store. Streamer 3 noted to us in the postsession survey that "The app currently comes off a bit shady, phones don't want to download it without extra permissions or turning off the 'don't download third party apps' function."
While viewers were able to capture and contribute sounds to the events they joined, we saw a consistent lag between contribution and validation which slowed down the process of getting sounds from the event into the game. The majority of support questions from streamers had to do with their viewers' sounds not being in the game. In response to these questions, a research team member explained that sounds must be validated before they could be used in the game. The research team member then explained that the validation interface of the app was on a separate screen from the streamer's event. Viewers would need to open the validation interface and confirm sounds before they would appear on stream. This prompted the streamers to explain the process to their viewers and encourage their viewers to validate sounds in the app, so that they could hear their sounds in the stream. Once viewers' sounds were validated they successfully appeared in the game and on the live stream.

\subsection{Managing Data Collection}

Although we had anticipated that streamers would encourage their viewers to collect sounds before the event, all of our streamers introduced the app and had viewers capture sounds during the streaming session. Streamers took different approaches to introducing their viewers to the data collection activity and managing their stream. Streamer $1 / 4$ introduced the app in the chat and verbally explained the data collection process on stream. During their first stream (S1), they set 10-minute time limits for their viewers to collect data. At the end of 10 minutes, the streamer then showed the sound collection leaderboard on stream and played the game. Over the course of three rounds of data collection and on-screen recognition, the streamer was able to progressively engage more viewers to collect and donate sounds to the stream. During their second stream (S4), the streamer used a similar strategy but made separate events in the data collection app for viewers to contribute sounds to.

Streamer 3 took a different approach and managed their stream in a less scheduled manner. The primary activity of their stream was the streamer crocheting. As they crocheted, they would interact continuously with the viewers to explain the game and the data collection process. The streamer would then play the game during breaks from their crochet work. The streamer also connected their phone to the live stream via screen sharing and directly demonstrated the app to their viewers. During the first two rounds of data 


\section{4 \\ Kitchen $\square$ Bathroom $\square$ Living $\square$ Garage $\square$ Ambience $\square$ Concerning $\square$ Unlabeled ${ }^{*}$}

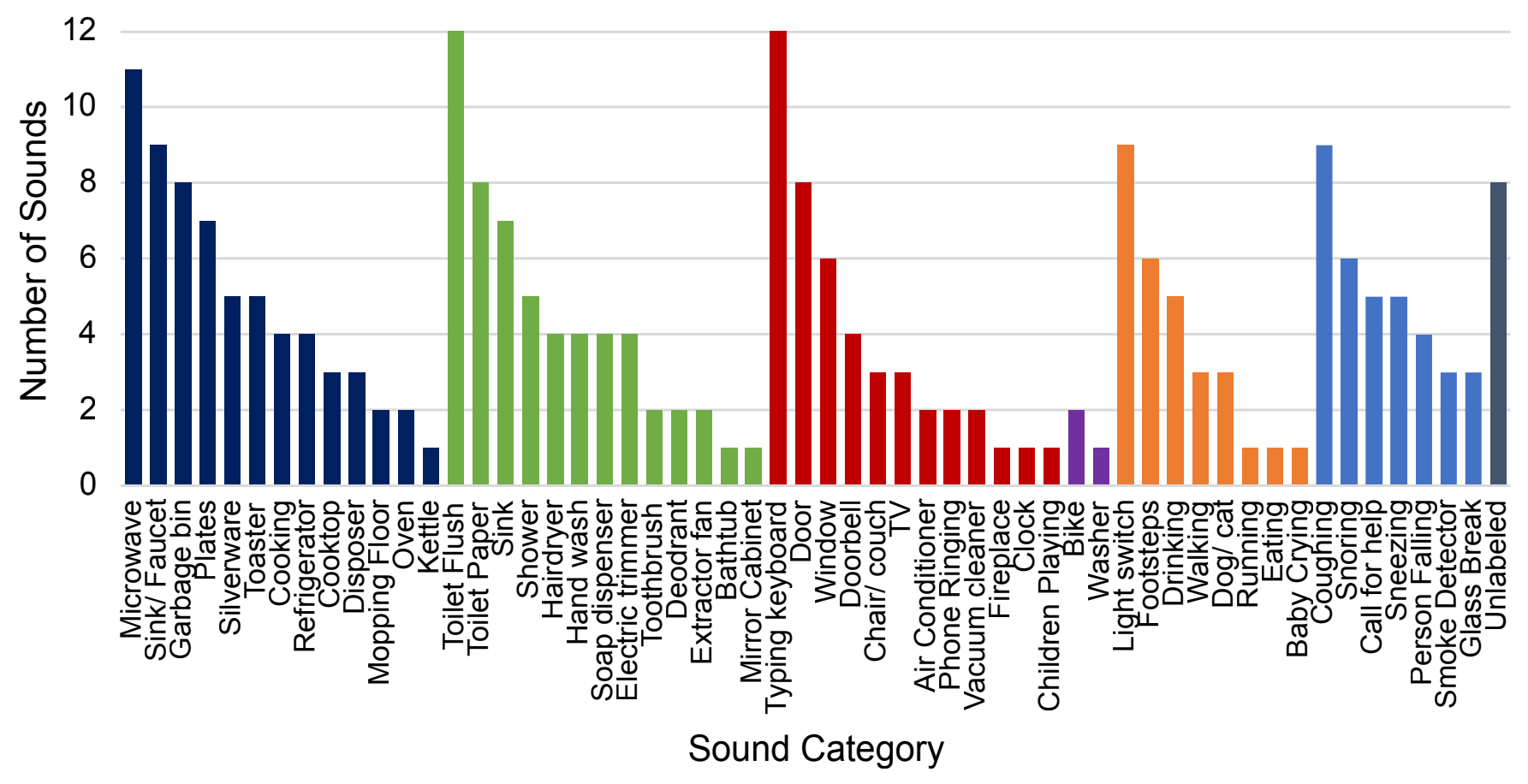

Figure 4: Total number of sounds collected across multiple categories. In total we collected and validated 212 sounds. Note that unlabeled sounds were from Stream 1 when we allowed viewers to upload their own sounds.

collection and gameplay, viewers collected sounds, but did not validate sounds. After realizing that their sounds were not used in the Rolling Rhapsody game, the streamer demonstrated the validation portion of the mobile app on screen. Figure 5 shows the number of sounds collected and validated over the course of the stream. A clear peak is shown right before the third game play session when the streamer rallied their viewers to validate sounds, leading to viewers validating 39 sounds in the span of five minutes.

\subsection{Viewer Engagement}

Over the course of the stream, we saw a rise in viewer participation in the form of more sounds being contributed and validated, as well as more comments in the stream chat discussing the data collection activity and Rolling Rhapsody game. As discussed above, viewers would often have a spike in participation when the streamer explicitly asked for sound capture and validation just before playing Rolling Rhapsody.

Viewer engagement was also positively influenced by the various recognition strategies used during the stream. In our observations during the stream, we saw that viewers hearing their own sounds and seeing their names shown on stream during gameplay lead to more participation in the stream chat. When viewers did not see their own sounds appear in the game, they would often make comments in the chat wondering why their sounds were not present. This then prompted the community to work together to collect and validate more sounds. For example, during one stream, when viewers discovered that their sounds were not being collected as often as sounds in the overall audio database, they coordinated with each other over chat to all choose the dragon avatar Then, they told the streamer to go collect dragons, increasing the chances that they would find the current viewers' sounds. Later, once the community had collected and validated enough sounds to fill the game, the viewers began picking different avatars (i.e. dragon, mosquito, mushroom) and would playfully send messages to the streamer to seek out their own avatar so that they could hear their sounds and get more recognition.

Viewers also responded well to the leaderboard, which was heavily used by Streamer $1 / 4$. For example, during the first round of data collection of Streamer 1's event, only 5 people participated in the sound collection process. However, after displaying the leaderboard on stream and showing that the top contributor collected 10 sounds, many of the viewers were impressed. Furthermore, when the streamer collected people's sounds, the viewers expressed in the chat that it was fun to hear other people's sounds. Overall, the motivation of the leaderboard encouraged more viewers to collect sounds in the later rounds of data collection and gameplay.

Another positive aspect of viewer engagement, seen in Stream 3 , was that the data collection activity engaged more of Streamer 3 's viewers than would normally engage. Streamer 3 reported that they often have 80-90 non-active viewers (people who watch but do not engage in the chat) on their streams but that only 2-3 people 


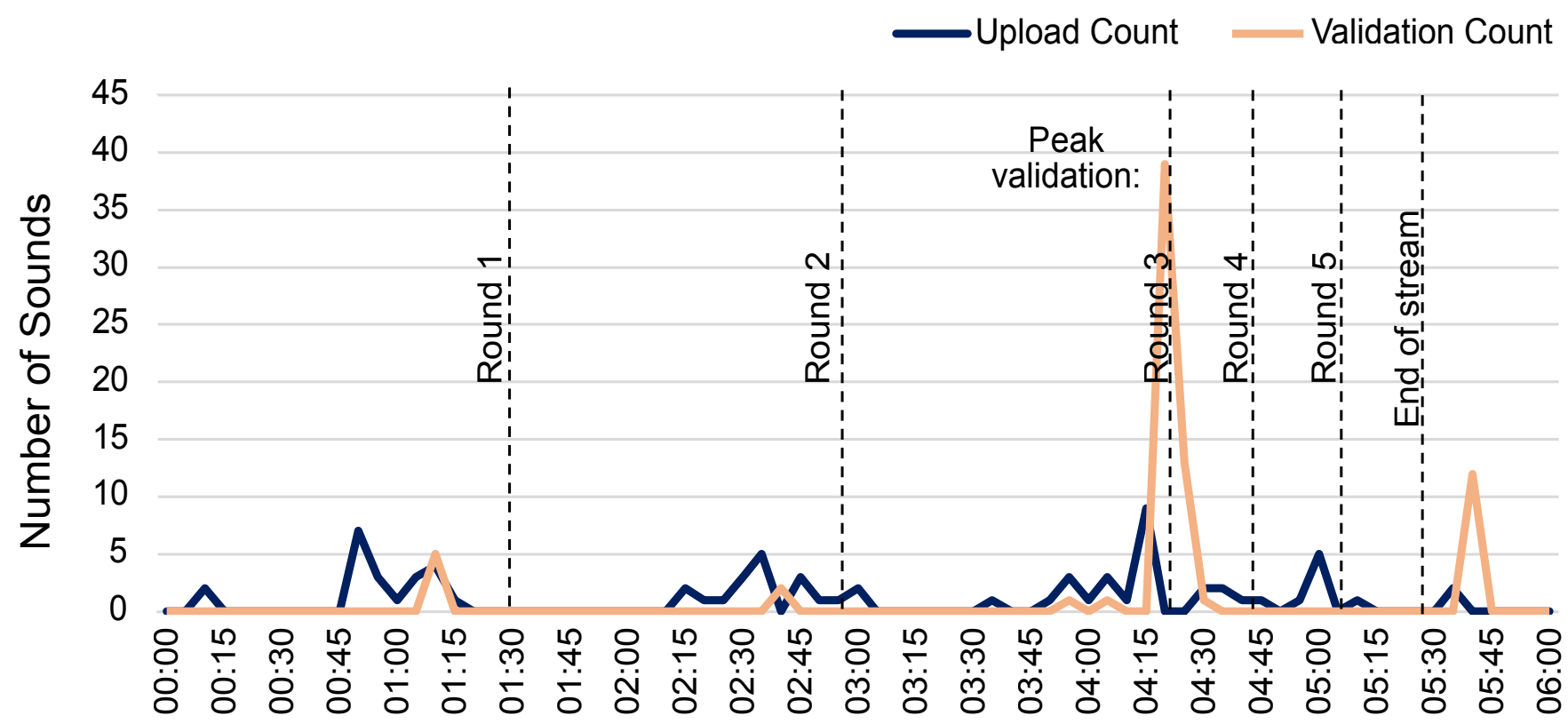

Time Since Start of Stream

Figure 5: Sounds uploaded and validated during Stream 3. Viewers collected sounds before each of 5 game play rounds, however, did not validate many sounds until rallied by the streamer before round 3.

typically interact with them. During the Polyphonic test session, 9 people actively participated in the chat and uploaded sounds.

Finally, another emergent way that viewers and streamers engaged with each other and with the data collection process was through negotiating what kinds of sounds to collect. During their second field test, Streamer 4 opened multiple events and negotiated which specific sound categories their audience wanted to play with (i.e. Bathroom sounds vs. Ambience) in each event. This engaged the viewers not just in collecting sounds, but also in being active in determining what kind of data should be collected, an interesting prospect for more participatory data collection activities and for potentially building longer term data collection relationships.

Overall, viewers were most excited to engage during three moments:

(1) When viewers' own sounds are played in-game, and their Twitch IDs are displayed on screen

(2) When the streamer actively requests that viewers take part in capture and validation activities

(3) When the streamer shows the leaderboard on screen and recognizes the top contributors

\subsection{Streamer Feedback}

In response to the questionnaire, we found that streamers had largely positive feedback concerning the concept of the game. All streamers rated the concept of audiences submitting audio that is then featured in the game as a positive aspect of the system. Streamers 3 and 4 both rated the game as engaging for their viewers and had favorable responses towards the simplicity of the app interface. However, one streamer commented that if the app were in an official app store, their audience would be more likely to trust it.

While one streamer appreciated the game concept saying that it can be used as a fun break between longer stream segments, another streamer commented that the game is fun, but has no clear goal for streamers or viewers which would compel them to continue playing. In response to this, they suggested that viewers be offered a larger incentive to collect sounds, such as unlocking new levels each time they collect a certain number of sounds.

Streamers 3 and 4 also agreed that the validation phase, although useful in helping streamers comply with Twitch community guidelines, is not integrated into the submission process well enough for app users to automatically understand that they need to validate sounds.

\section{DISCUSSION}

Through our field tests, we demonstrate that our proof-of-concept allows streamers to engage their audiences in collecting audio data from the home. We find that many of our design decisions, such as the active use of viewers' sounds in the live gameplay and the recognition provided to viewers, can foster positive engagement between the streamers and viewers and within the viewer community itself. Given our results, we discuss some of the more surprising findings related to data being collected during the live stream sessions, challenges with our split between data collection and validation, the role of our game and data collection activities in non-gaming live streams, and possible avenues and design implications for future development of long-term data collection relationships on live streaming platforms. 


\subsection{Timing of data collection}

As described above, we were surprised by the timing of data collection. Viewers did not collect sounds outside of the stream itself. Instead, streamers directed them to step away from the stream and collect sounds while the streamer was present. Our very first designs relied on viewers collecting sounds during a stream, as streamers told us that they wanted times when they could step back from constant performance. However, early playtests convinced us that streamers were worried about viewers losing interest. At the same time, we knew that viewers participate in stream-related activities, such as hanging out on Discord, before and after the stream itself [44]. Following our design principle of meeting streamers' needs, we adjusted the focus of our design to explicitly support collecting data before the stream - and were surprised to see that the behavior we had planned for did not appear.

One hypothesis for this difference has to do with stream size. Our playtests targeted relatively small streamers, with 6-1000 regular viewers [14]. At this stream size, streamers can have ongoing relationships and conversations with their viewers; at the same time, they may be less likely to have a Discord or other dedicated out-of-stream space for interaction, both because the viewership may not be enough to support it and because the stream chat itself can serve a relationship-building function. For these smaller streams, on-stream activities may edge out out-of-stream activities.

We also observed that streamers were actively engaged with the social coordination aspect of data collection. In order to honor viewer contributions, we had designed on-screen recognition activities to reward both individual and community accomplishments (e.g. the word cloud). However, streamers pushed hard toward community recognition. They made sound capture an activity that the whole stream was doing together, even though they were physically separated and collecting different sounds. As part of this process, streamers helped manage viewer attention [51], explicitly telling their viewers when to pay attention to the data collection task in their home and when to pay attention to the stream. As a result, activities that streamers incorporated into their activity flow got high engagement from viewers (as shown in the data spikes in Figure 5). Conversely, portions of the experience that did not get incorporated into the streamer's activity flow, such as validation, were sticking points. See below for a fuller discussion.

What are the implications of this finding? First, by designing for flexibility, we can accommodate streamers with different size streams. Even when we hypothesized that viewers would collect and validate sounds before the stream, we didn't require them to do so. Similarly, although we found that viewers captured sounds during the stream, we should not remove the capacity to collect sounds outside of stream-time. Our modular approach to design supports this flexibility; for example, future work in streams with different social expectations and structure might use different data collection modules. Second, we need to design for streamers' role as social coordinators. Data capture happened during stream-time because that is when our streamers chose to coordinate the activity. However, streamers often coordinate social activities outside the stream, even if it is as simple as recruiting viewers to come watch them. If we are to design out-of-stream activities, we can incorporate streamers as activity coordinators, instead of trying to design the system to motivate all interactions without direct intervention from the streamer.

\subsection{Challenges motivating validation}

Data validation was an ongoing challenge for us. Unlike data capture, the question was not the timing of the activity, but whether viewers were participating at all. Both streamers and viewers were uncertain where it fit into the larger game framework. For example, they expressed confusion when the sounds they had collected were not appearing in the game. Viewers validated sounds only when told by the streamer that sounds had to be validated before showing up on stream.

Why did this happen? In part, it may be a side effect of data collection happening during the stream. Viewers don't have time to play around with the app and explore its various features when under time pressure. Instead, viewers followed streamers' instructions. If the streamer did not direct them to a feature, they may have been less likely to use it.

Additionally, we also see this as part of a larger design issue. As we noted in our description of the design, the focus of our first prototype was on capturing sounds. Our design decisions were about rewarding viewers for the sounds they collected, and motivating streamers to encourage their viewers to participate in data capture. In our design, however, validation is a "gating" activity - a task that sounds have to go through before they can be used on stream. Furthermore, in the app, the validation activity is not associated with the streamer's event. Instead, the validation portion was a secondary interface accessed from the app's home screen and was for all sounds across any events using Polyphonic. In other words, we failed to live up to our original design principle of modular design where every component has a clear purpose and provides clear value to the stakeholders. Doing so would have meant treating validation as a "first-class" activity - one where our playful and social rewards are directed at the act of identifying and validating sounds.

Returning to our design documentation with this in mind, we realized that the problem we were actually trying to solve with our validation strategy was a moderation problem. We did not want viewers to use their sound contributions to harass the streamer, or one another, or to violate the platform's terms of service. At the same time, we wanted to incentivize viewers to label sounds correctly. The person doing the capture knew what was making the sound in question; however, identifying a sound might be very difficult later. We did not want viewers to mislabel their sounds. We also anticipated that viewers could collect and send deliberately incorrect sounds, such as sounds of people talking or obscenities. Requiring validation before sounds could appear on-stream would solve both these problems at once.

We now recognize that conflating moderation, labeling, and validation was confusing for viewers. Additionally, our failure to integrate it into the on-stream activity pipeline and provide clear value during the validation process meant that viewers often did not even recognize that validation was a relevant activity. To address this issue in future designs, we can re-investigate some of our early design ideas for addressing sound moderation. For example, channel moderators can be a part of this process. Collected 
sounds can go into a queue and moderators can filter out all the sounds that are inappropriate. We also discovered that for home audio data collection, automated filtering was surprisingly effective. Filtering out anything with a voice in it-which is required by our consent process in any case-enormously reduces the options for harassment or other bad behavior. Finally, we can explore modular designs for validation processes that can stand on their own as entertaining activities. This might include designing the validation process as a mini-game, or designing explicit on-stream features and recognition associated with the validation process.

\subsection{Games vs. gamers}

Starting early in our process, we conceptualized Twitch as a game streaming platform. While we knew that other types of activities take place on Twitch [14], we expected that our proof-of-concept would largely appeal to people who already use the platform to play games. However, our most engaged streamer was Streamer 3 , who runs a craft stream. They engaged for substantially longer than any other streamer. While it is obviously a small sample, Streamer 3's experience suggests that it is at least possible for non-game streamers to be deeply engaged with our experience, even though we were not explicitly designing for them.

In order to follow through on our design principle of meeting streamers' needs, we must understand why Streamer 3 found our experience so compelling. Non-game streamers share many of the same needs as game streamers. They want an engaged audience, they want to grow their audiences, and they want to build connections with the audiences they have. Our proof-of-concept is designed around these underlying needs, not around any specific game activity.

At the same time, our game and recognition activities may actually benefit from the contrast with non-game activities. Community expectations about game polish are different. We believe that Rolling Rhapsody benefited from being contrasted with a completely different activity, instead of with professional game studios' work. Additionally, the data collection and game interactions could be used as a break activity in non-gaming streams aimed explicitly at building community engagement, without being in competition with the "real" gaming content of a channel. While games are the largest group of streams on Twitch, other major categories such as Creative, Just Chatting and IRL have become exceedingly popular and represent some of the most watched streams [6, 14], significantly opening up our potential pool of streamers to partner with and viewers to engage. Designing experiences that can be used as breaks can also help our larger design goal of sustainability, as the activity becomes a part of the culture of the stream over time rather than a one-off game.

\subsection{Envisioning the future}

For our field tests, we asked streamers to play for at least 30 minutes. However, all three streamers engaged for substantially longer than the study required during their test events. For example, Streamer 3 returned to the game five times over the course of 5.5 hours, while Streamer 1 requested access to the game so that they could play it in another streaming session, which they did as Streamer 4. This behavior is, to say the least, unusual for research participants. It would be flattering to attribute it to the excellence of our design. However, we think it is more likely to be related to aspects of the Twitch ecosystem.

Why long sessions? The average viewer watches for 30-60 minutes, but viewers also drop in and out. They might not arrive at the beginning of the stream. So long sessions (to engage viewers while they are there) with periodic dips into the Polyphonic ecosystem (since a different subset of viewers will be there each time) makes strategic sense. Why replay? Once the streamer has sunk the cost of getting the Polyphonic system set up, it makes sense to maximize the number of viewers it engages. Just as viewers drop in and out of a single session, different viewers join different streaming sessions. All of our streamers had significantly more followers than they did active viewers. By replaying the game and participating in data collection activities over time, the streamer can engage viewers who haven't seen the game before, while viewers who know what they're doing can feel like experts within their community. These desires align with what we know about why people value streaming [51].

What these patterns suggest is that Polyphonic can become an opportunity for long-term data collection relationships. If streamers are willing to engage longitudinally across time, and viewers repeatedly come back to their stream, we can engage the same people over and over again instead of recruiting new folks every time. This would let us collect novel and robust datasets, like the sound of the same child crying every day as they age, which in turn would let us ask new data-driven research questions.

The other thing that this long-term engagement would enable is to more deeply engage the community in the data collection process. During Stream 4 we found preliminary evidence of this with the streamer negotiating with their community to decide what categories of sounds to collect. Research on citizen science shows that it is possible to work with communities at a variety of levels, from simply gathering data to having them be collaborators on scientific enterprises, and that operating at these various levels can improve engagement in the scientific tasks [46]. If streamers and viewers are willing to engage long-term, that gives them the kind of stake that, we hope, can be built into a collaborative relationship around data.

\subsection{Design Implications}

We can conceptualize our insights from this study around our four original design principles. Based on these, we suggest design implications and future work for live stream based data collection activities.

6.5.1 Respect streamers' needs. Streamers have common needs, even across apparently divergent categories like game streaming and crafts streaming. They want to engage their audiences and grow their communities. While community-oriented data collection activities may serve as a way to engage their audiences, the scale and structure of their communities may affect how streamers can meet these needs. For example, we think that our field test streamers focused on collecting data in real-time due to the size and character of their community, with more focus put onto in-stream interaction as opposed to out-of-stream activities. The implication here is that designers looking to create data collection activities 
alongside streamers should characterize streamers' resources and strategies for community engagement as well as their needs. Future work might consider new tools to capture such strategies and support designers in creating activities that better match individual streams. Work from Wohn \& Freeman [60] can provide information that designers can use to better tailor their experiences around the different strategies that streams use to engage their audiences.

6.5.2 Respect viewers' contributions. We originally framed this as a need for our team to respect viewer contributions in the data collection system and game design. In practice, however, we found that viewers were more interested in gaining respect from the streamer, either as individuals or as a collective. Our designs for on-screen recognition and a leaderboard seem to been well-received due to the additional recognition given by the streamer using these design elements. An implication here is that future system designs may focus their recognition features around assisting the streamer to express appreciation, recognition, and respect towards their viewers. More consideration should be given to crafting on- and offstream recognition features such as in-game graphics, leaderboards, or chat systems that invite the streamer to personally recognize viewers, helping to support viewers need for recognition in the community. One limitation of our current design work is that while we did include streamers in our design process and feedback, we did not include viewers. Future design work should look to include both streamers and viewers together in order to better serve the needs of viewers in crafting positive data collection relationships.

6.5.3 Build a modular system. In our struggles with sound validation, we found that while our systems were developed to be technically separable, we did not fully achieve our goal of developing modules that each serve a clear purpose and provide direct value during the various ways that streamers might engage their viewers through the data collection and validation process. Originally, we conceived of validation as happening before the stream and as part of a larger data collection effort across streams. Instead, future design should consider allowing streamers to run different modules independently and in any order-while still giving them a way to feel ownership of the activities. If viewers are not collecting "my stream's sounds," that sense of ownership might need to look very different that what we have currently developed. For example, in a segmentation activity, viewers might get sounds from the database based on a query the stream collectively selects. Finding these alternate forms of connection and ownership will be an important design direction going forward.

6.5.4 Build a sustainable system. Based on our proof-of-concept deployment, we see substantial potential for long-term engagement within data collection systems developed around live streaming communities. Across our field test sessions we found that streamers employed the system in ways that helped promote positive relationships among their communities. Furthermore, we have found preliminary evidence that such data collection activities can weave into a stream's other content and can be repeated. To build on this, future designs might look to further engage with interspersed data collection activities by employing principles of snackable gameplay [11]. While our original thought was to focus on replayability, snackability may add new options for systems designers to employ such as enforced waiting periods that ask streamers to return to the game rather than continue with the game. Additionally, in order to create sustainable systems for real-world data collection, it will also be necessary to scale up to larger numbers of contributors. While our work shows a first of its kind system, more research and design work is needed to understand how such stream-based data collection activities can engage hundreds or thousands of viewers. Such work may require new game designs that allow more viewers' content to contribute to the gameplay and new recognition strategies that keep large numbers of viewers motivated.

\section{BARRIERS AND LIMITATIONS}

Although our field tests have provided preliminary evidence that our proof-of-concept can engage live streaming communities in data collection tasks, we find that there are barriers and limitations that can limit participation in these activities. These limitations of the system can be divided into three main themes.

First are the barriers and limitations presented by the technology associated with the system. There is the expectation that in order to participate in the game, one has a certain level of technical literacy so that the viewer can easily download the mobile app, record sounds, access the Twitch stream, and engage with other viewers in the community. Furthermore, participants would need to have access to high-speed internet and mobile devices so that they can use the app and access the Twitch stream. In the proof-ofconcept's current state, without these two essential prerequisites, it is impossible to contribute.

Second, there may be limitations in who engages in the data collection activities. Because Twitch emphasizes gaming communities, our population of data collectors is likely to be different from those we could recruit through other data collection platforms. Twitch caters to an audience that is $65 \%$ male, under 34 years of age, and primarily from the US, Europe, and Russia [1]. Furthermore, we note that there is an age limitation on who can use the app. Only legal adults (18+) can consent to share audio data, so a significant portion of the Twitch community is excluded from participating in audio collection. This being said, with the growth of non-gaming streams, more and more people are tuning into Twitch, possibly opening up new viewer populations as time progresses.

Third, there are currently accessibility barriers that may prevent those of different abilities from participating in our data collection activities. One of the biggest barriers of our proof-of-concept is to low-hearing and deaf participants. Because the proof-of-concept relies so heavily on the collection of audio data, it would be challenging for these segments of the population to participate even if they have a presence on Twitch.

\subsection{Ethical Implications}

As more work is done exploring how to engage live streaming communities in data collection tasks, there are ethical implications that should be considered, especially as such systems scale to larger numbers of viewers. Three areas for consideration include privacy and anonymity around data collection, labor ethics in regards to volunteer work, and the power dynamics between streamers and viewers. 
In our proof-of-concept deployment, we explicitly recognized viewer contributions on-screen, motivated by prior work around recognition in citizen science and live streaming communities. While this method of recognition is common among Twitch streams and many viewers may appreciate the recognition, this does associate the data with an individual viewer. While many smaller streaming communities may already know other viewers by screen name and enjoy this kind of interaction, some viewers may not want to be associated with their exact data (i.e. the sounds they recorded). Future work should explore different strategies for recognition that also consider viewers' privacy preferences. While prior work has found that many citizen scientists are happy to share their data to support scientific efforts [5], care should be taken to minimize any personally identifying information collected during data collection tasks such as geolocation or images of people without proper consent.

In regards to labor ethics, working with live streaming viewers to collect and annotate data may have similar challenges to other forms of crowdwork in regards to alienating workers through poor communication and non-compensated work [26]. While we have designed our proof-of-concept to provide non-monetary benefits and an enjoyable experience, there is still a risk that viewers and streamers may not receive the benefits that we are proposing. Furthermore, data requesters may leverage such a system for more free labor, though our intent is to provide an alternative strategy. Future research should consider and explore alternative strategies for compensation and similar systems should develop effective communication between data requesters, streamers, and workers to facilitate positive data collection relationships [26].

Finally, due to the parasocial relationship between streamers and viewers, care should be given on what kinds of data are collected and how streamers might encourage their viewers. Streamers might compel viewers to collect more personal data due to the power dynamics of the streamer viewer relationship. Strategies should be explored to balance motivating viewers without making them feel coerced.

\section{CONCLUSION}

In this paper, we explored how we can leverage the social interactions and positive relationships between live streamers and their viewers to facilitate data collection of audio in the home as a novel alternative to more traditional crowdwork data collection strategies. We developed a proof-of-concept system, Polyphonic, to engage these live streaming communities in data collection and built our system around a set of design principles based on crowdwork, citizen science, and live streaming literature and the direct needs of streamers and viewers. Using a participatory game, mobile data collection app, and various recognition strategies for data contribution, we showed that live stream communities can collect data while also fostering positive engagement between the streamer and their viewers. Our field tests revealed that for such a data collection system, the streamer plays a critical role in coordinating the data collection activities during the stream, suggesting a future for activities where viewers watch the stream and participate in community activities away from the screen. Our work has implications for new ways to conduct citizen science and data collection with live streaming communities. Furthermore, our work suggests possible design opportunities for developing activities that can facilitate long-term relationships between streamers and their viewers.

\section{ACKNOWLEDGMENTS}

We would like to thank our steamers and their viewers for participating in our field tests. We would also like to acknowledge the efforts of our student development team including Yifeng Shi, Charlie Li, Ann Maria Jose, Yikai Han, Yifan Deng, Aishwarya Jaiswal, Sanya Agrawal, Freya Mi, Andrew Yu, and Mingzhi Cai. This work was supported by a gift from Phillips and Bosch.

\section{REFERENCES}

[1] 2020. Twitch Revenue and Usage Statistics (2020). https://www.businessofapps. com/data/twitch-statistics/

[2] Bethany Alender. 2016. Understanding volunteer motivations to participate in citizen science projects: a deeper look at water quality monitoring. Fournal of Science Communication 15, 3 (2016), A04.

[3] Michael G Blight. 2016. Relationships to video game streamers: Examining gratifications, parasocial relationships, fandom, and community affiliation online. (2016).

[4] Rick Bonney, Caren B Cooper, Janis Dickinson, Steve Kelling, Tina Phillips, Kenneth V Rosenberg, and Jennifer Shirk. 2009. Citizen science: a developing tool for expanding science knowledge and scientific literacy. BioScience 59, 11 (2009), 977-984.

[5] Anne Bowser, Katie Shilton, Jenny Preece, and Elizabeth Warrick. 2017. Accounting for Privacy in Citizen Science: Ethical Research in a Context of Openness. In Proceedings of the 2017 ACM Conference on Computer Supported Cooperative Work and Social Computing (Portland, Oregon, USA) (CSCW '17). Association for Computing Machinery, New York, NY, USA, 2124-2136. https: //doi.org/10.1145/2998181.2998305

[6] Chase. 2021. State of the Stream December and 2020 Year in Review. https://blog.streamelements.com/state- of-the-stream-december-and2020-year-in-review-aa4146f074be

[7] Honglie Chen, Weidi Xie, Andrea Vedaldi, and Andrew Zisserman. 2020. Vggsound: A large-scale audio-visual dataset. In ICASSP 2020-2020 IEEE International Conference on Acoustics, Speech and Signal Processing (ICASSP). IEEE, 721-725.

[8] Seth Cooper, Firas Khatib, Adrien Treuille, Janos Barbero, Jeehyung Lee, Michael Beenen, Andrew Leaver-Fay, David Baker, Zoran Popović, et al. 2010. Predicting protein structures with a multiplayer online game. Nature 466, 7307 (2010), 756-760.

[9] Vickie Curtis. 2015. Motivation to participate in an online citizen science game: A study of Foldit. Science Communication 37, 6 (2015), 723-746.

[10] Nils Dahlbäck, Arne Jönsson, and Lars Ahrenberg. 1993. Wizard of Oz Studies: Why and How. In Proceedings of the 1st International Conference on Intelligent User Interfaces (IUI '93). ACM, New York, NY, USA, 193-200. https://doi.org/10. $1145 / 169891.169968$

[11] Drew Davidson. [n.d.]. Snackable Gameplay. https://waxebb.com/writings/ snackgame.html

[12] Jia Deng, Wei Dong, Richard Socher, Li-Jia Li, Kai Li, and Li Fei-Fei. 2009. Imagenet: A large-scale hierarchical image database. In 2009 IEEE conference on computer vision and pattern recognition. Ieee, 248-255.

[13] Nathan Eagle. 2009. txteagle: Mobile crowdsourcing. In International Conference on Internationalization, Design and Global Development. Springer, 447-456.

[14] Claudia Flores-Saviaga, Jessica Hammer, Juan Pablo Flores, Joseph Seering, Stuart Reeves, and Saiph Savage. 2019. Audience and Streamer Participation at Scale on Twitch. In Proceedings of the 30th ACM Conference on Hypertext and Social Media. 277-278.

[15] Tracy Fullerton. 2014. Game design workshop: a playcentric approach to creating innovative games. CRC press.

[16] Jort F Gemmeke, Daniel PW Ellis, Dylan Freedman, Aren Jansen, Wade Lawrence, R Channing Moore, Manoj Plakal, and Marvin Ritter. 2017. Audio set: An ontology and human-labeled dataset for audio events. In 2017 IEEE International Conference on Acoustics, Speech and Signal Processing (ICASSP). IEEE, 776-780.

[17] William A. Hamilton, Oliver Garretson, and Andruid Kerne. 2014. Streaming on Twitch: Fostering Participatory Communities of Play within Live Mixed Media. Association for Computing Machinery, New York, NY, USA, 1315-1324. https: //doi-org.proxy.library.cmu.edu/10.1145/2556288.2557048

[18] Kotaro Hara, Abigail Adams, Kristy Milland, Saiph Savage, Chris Callison-Burch, and Jeffrey P Bigham. 2018. A data-driven analysis of workers' earnings on Amazon Mechanical Turk. In Proceedings of the 2018 CHI Conference on Human Factors in Computing Systems. 1-14. 
[19] Erik Harpstead, Juan Sebastian Rios, Joseph Seering, and Jessica Hammer. 2019. Toward a Twitch Research Toolkit: A Systematic Review of Approaches to Research on Game Streaming. In Proceedings of the Annual Symposium on Computer-Human Interaction in Play (Barcelona, Spain) (CHI PLAY '19). Association for Computing Machinery, New York, NY, USA, 111-119. https: //doi.org/10.1145/3311350.3347149

[20] Yurong He, Julia K Parrish, Shawn Rowe, and Timothy Jones. 2019. Evolving interest and sense of self in an environmental citizen science program. Ecology and Society 24, 2 (2019).

[21] Zorah Hilvert-Bruce, James T Neill, Max Sjöblom, and Juho Hamari. 2018. Social motivations of live-streaming viewer engagement on Twitch. Computers in Human Behavior 84 (2018), 58-67.

[22] Mansoor Iqbal. 2020. Twitch Revenue and Usage Statistics (2020). Business of Apps (Oct 2020). https://www.businessofapps.com/data/twitch-statistics/

[23] Mark R Johnson and Jamie Woodcock. 2019. “And today's top donator is”: How live streamers on Twitch. tv monetize and gamify their broadcasts. Social Media+ Society 5, 4 (2019), 2056305119881694.

[24] Rebecca Jordan, Alycia Crall, Steven Gray, Tina Phillips, and David Mellor. 2015 Citizen science as a distinct field of inquiry. Bioscience 65, 2 (2015), 208-211.

[25] Anna Kasunic, Chun-Wei Chiang, Geoff Kaufman, and Saiph Savage. 2019. Turker Tales: Integrating Tangential Play into Crowd Work. In Proceedings of the 2019 on Designing Interactive Systems Conference. 21-34.

[26] Aniket Kittur, Jeffrey V. Nickerson, Michael Bernstein, Elizabeth Gerber, Aaron Shaw, John Zimmerman, Matt Lease, and John Horton. 2013. The Future of Crowd Work. In Proceedings of the 2013 Conference on Computer Supported Cooperative Work (CSCW '13). ACM, New York, NY, USA, 1301-1318. https://doi.org/10. $1145 / 2441776.2441923$

[27] Joon Soo Lim, Min-Ji Choe, Jun Zhang, and Ghee-Young Noh. 2020. The role of wishful identification, emotional engagement, and parasocial relationships in repeated viewing of live-streaming games: A social cognitive theory perspective. Computers in Human Behavior (2020), 106327.

[28] Chris J Lintott, Kevin Schawinski, Anže Slosar, Kate Land, Steven Bamford, Daniel Thomas, M Jordan Raddick, Robert C Nichol, Alex Szalay, Dan Andreescu, et al 2008. Galaxy Zoo: morphologies derived from visual inspection of galaxies from the Sloan Digital Sky Survey. Monthly Notices of the Royal Astronomical Society 389, 3 (2008), 1179-1189.

[29] Colleen Macklin and John Sharp. 2016. Games, Design and Play: A detailed approach to iterative game design. Addison-Wesley Professional.

[30] Andrew Mao, Ece Kamar, Yiling Chen, Eric Horvitz, Megan E Schwamb, Chris J Lintott, and Arfon M Smith. 2013. Volunteering versus work for pay: Incentives and tradeoffs in crowdsourcing. In First AAAI conference on human computation and crowdsourcing. Citeseer.

[31] David Martin, Benjamin V Hanrahan, Jacki O'Neill, and Neha Gupta. 2014. Being a turker. In Proceedings of the 17th ACM conference on Computer supported cooperative work \& social computing. 224-235.

[32] Ankit Mittal and Donghee Yvette Wohn. 2019. Charity Streaming: Why Charity Organizations Use Live Streams for Fundraising. In Extended Abstracts of the Annual Symposium on Computer-Human Interaction in Play Companion Extended Abstracts. 551-556.

[33] Jędrzej Olejniczak. 2015. A linguistic study of language variety used on twitch. tv: desriptive and corpus-based approaches. Redefining Community in Intercultural Context 4, 1 (2015), 329-334.

[34] Katherine Payne, Mark J Keith, Ryan M Schuetzler, and Justin Scott Giboney 2017. Examining the learning effects of live streaming video game instruction over Twitch. Computers in Human Behavior 77 (2017), 95-109.

[35] Anthony J Pellicone and June Ahn. 2017. The Game of Performing Play: Understanding streaming as cultural production. In Proceedings of the $2017 \mathrm{CHI}$ Conference on Human Factors in Computing Systems. 4863-4874.

[36] Tina B Phillips, Heidi L Ballard, Bruce V Lewenstein, and Rick Bonney. 2019. Engagement in science through citizen science: Moving beyond data collection. Science Education 103, 3 (2019), 665-690.

[37] M Jordan Raddick, Georgia Bracey, Pamela L Gay, Chris J Lintott, Phil Murray, Kevin Schawinski, Alexander S Szalay, and Jan Vandenberg. 2009. Galaxy zoo: Exploring the motivations of citizen science volunteers. arXiv preprint arXiv:0909.2925 (2009).

[38] Dana Rotman, Jenny Preece, Jen Hammock, Kezee Procita, Derek Hansen, Cynthia Parr, Darcy Lewis, and David Jacobs. 2012. Dynamic changes in motivation in collaborative citizen-science projects. In Proceedings of the ACM 2012 conference on computer supported cooperative work. 217-226.

[39] Olga Russakovsky, Jia Deng, Hao Su, Jonathan Krause, Sanjeev Satheesh, Sean Ma, Zhiheng Huang, Andrej Karpathy, Aditya Khosla, Michael Bernstein, et al 2015. Imagenet large scale visual recognition challenge. International journal of computer vision 115,3 (2015), 211-252.

[40] Saiph Savage, Andres Monroy-Hernandez, and Tobias Höllerer. 2016. Botivist Calling Volunteers to Action Using Online Bots (CSCW '16). Association for Computing Machinery, New York, NY, USA. https://doi.org/10.1145/2818048. 2819985
[41] Joseph Seering. [n.d.]. Twitch Bibliography. https://docs.google.com/ spreadsheets/d/1s1jyvJR3K_b8FmH1X-U_Xa6EP1Od2iAIOgaz98b2_A0/edit\# gid $=0$

[42] Joseph Seering, Michal Luria, Connie Ye, Geoff Kaufman, and Jessica Hammer. 2020. It Takes a Village: Integrating an Adaptive Chatbot into an Online Gaming Community. In Proceedings of the 2020 CHI Conference on Human Factors in Computing Systems (Honolulu, HI, USA) (CHI '20). Association for Computing Machinery, New York, NY, USA, 1-13. https://doi.org/10.1145/3313831.3376708

[43] Joseph Seering, Saiph Savage, Michael Eagle, Joshua Churchin, Rachel Moeller, Jeffrey P Bigham, and Jessica Hammer. 2017. Audience participation games: Blurring the line between player and spectator. In Proceedings of the 2017 Conference on Designing Interactive Systems. 429-440.

[44] Jeff T Sheng and Sanjay R Kairam. 2020. From Virtual Strangers to IRL Friends: Relationship Development in Livestreaming Communities on Twitch. Proceedings of the ACM on Human-Computer Interaction 4, CSCW2 (2020), 1-34.

[45] Stephen Tsung-Han Sher and Norman Makoto Su. 2019. Speedrunning for Charity: How Donations Gather Around a Live Streamed Couch. Proceedings of the ACM on Human-Computer Interaction 3, CSCW (2019), 1-26.

[46] Jennifer L Shirk, Heidi L Ballard, Candie C Wilderman, Tina Phillips, Andrea Wiggins, Rebecca Jordan, Ellen McCallie, Matthew Minarchek, Bruce V Lewenstein, Marianne E Krasny, et al. 2012. Public participation in scientific research: a framework for deliberate design. Ecology and society 17, 2 (2012).

[47] Gunnar A Sigurdsson, Gül Varol, Xiaolong Wang, Ali Farhadi, Ivan Laptev, and Abhinav Gupta. 2016. Hollywood in homes: Crowdsourcing data collection for activity understanding. In European Conference on Computer Vision. Springer, 510-526.

[48] M. S. Silberman, B. Tomlinson, R. LaPlante, J. Ross, L. Irani, and A. Zaldivar. 2018. Responsible Research with Crowds: Pay Crowdworkers at Least Minimum Wage. Commun. ACM 61, 3 (Feb. 2018), 39-41. https://doi.org/10.1145/3180492

[49] Robert Simpson, Kevin R. Page, and David De Roure. 2014. Zooniverse: Observing the World's Largest Citizen Science Platform. In Proceedings of the 23rd International Conference on World Wide Web (Seoul, Korea) (WWW'14 Companion). Association for Computing Machinery, New York, NY, USA, 1049-1054. https://doi.org/10.1145/2567948.2579215

[50] Push Singh, Thomas Lin, Erik T Mueller, Grace Lim, Travell Perkins, and Wan Li Zhu. 2002. Open mind common sense: Knowledge acquisition from the general public. In OTM Confederated International Conferences" On the Move to Meaningful Internet Systems". Springer, 1223-1237.

[51] Max Sjöblom and Juho Hamari. 2017. Why do people watch others play video games? An empirical study on the motivations of Twitch users. Computers in human behavior 75 (2017), 985-996.

[52] Alexander Sorokin and David Forsyth. 2008. Utility data annotation with amazon mechanical turk. In 2008 IEEE computer society conference on computer vision and pattern recognition workshops. IEEE, 1-8.

[53] Hao Su, Jia Deng, and Li Fei-Fei. 2012. Crowdsourcing annotations for visual object detection. In Workshops at the Twenty-Sixth AAAI Conference on Artificial Intelligence.

[54] Brian L Sullivan, Christopher L Wood, Marshall J Iliff, Rick E Bonney, Daniel Fink, and Steve Kelling. 2009. eBird: A citizen-based bird observation network in the biological sciences. Biological conservation 142, 10 (2009), 2282-2292.

[55] TL Taylor. 2018. Twitch and the Work of Play. American fournal of Play 11, 1 (2018), 65-84.

[56] Luis Von Ahn and Laura Dabbish. 2005. ESP: Labeling Images with a Computer Game.. In AAAI spring symposium: Knowledge collection from volunteer contributors, Vol. 2.

[57] Luis Von Ahn and Laura Dabbish. 2008. Designing games with a purpose. Commun. ACM 51, 8 (2008), 58-67.

[58] Yufeng Wang, Xueyu Jia, Qun Jin, and Jianhua Ma. 2017. Mobile crowdsourcing: framework, challenges, and solutions. Concurrency and Computation: Practice and experience 29, 3 (2017), e3789.

[59] Yiwei Wang, Nicole Kaplan, Greg Newman, and Russell Scarpino. 2015. CitSci. org: A new model for managing, documenting, and sharing citizen science data. PLoS Biol 13, 10 (2015), e1002280.

[60] Donghee Yvette Wohn and Guo Freeman. 2020. Audience management practices of live streamers on Twitch. In ACM International Conference on Interactive Media Experiences. 106-116.

[61] Donghee Yvette Wohn and Guo Freeman. 2020. Live Streaming, Playing, and Money Spending Behaviors in eSports. Games and Culture 15, 1 (2020), 73-88.

[62] Donghee Yvette Wohn, Guo Freeman, and Caitlin McLaughlin. 2018. Explaining viewers' emotional, instrumental, and financial support provision for live streamers. In Proceedings of the 2018 CHI conference on human factors in computing systems. 1-13.

[63] Jamie Woodcock and Mark R Johnson. 2019. The affective labor and performance of live streaming on Twitch. tv. Television \& New Media 20, 8 (2019), 813-823.

[64] Jason Wu, Chris Harrison, Jeffrey P. Bigham, and Gierad Laput. 2020. Automated Class Discovery and One-Shot Interactions for Acoustic Activity Recognition. In Proceedings of the 2020 CHI Conference on Human Factors in Computing Systems (Honolulu, HI, USA) (CHI '20). Association for Computing Machinery, New York, 
DIS '21, June 28-July 2, 2021, Virtual Event, USA

NY, USA, 1-14. https://doi.org/10.1145/3313831.3376875

[65] Tingxin Yan, Matt Marzilli, Ryan Holmes, Deepak Ganesan, and Mark Corner 2009. MCrowd: A Platform for Mobile Crowdsourcing. In Proceedings of the 7th ACM Conference on Embedded Networked Sensor Systems (Berkeley, California) (SenSys '09). Association for Computing Machinery, New York, NY, USA, 347-348.
Nikolas Martelaro, Tarannum Lakdawala, Jingya Chen, and Jessica Hammer

https://doi.org/10.1145/1644038.1644094

[66] John Zimmerman and Jodi Forlizzi. 2019. Service Design. https: //www.interaction-design.org/literature/book/the-encyclopedia-of-humancomputer-interaction-2nd-ed/service-design 